\title{
Enhancing the Teaching and Learning Interpretation of Box-and-Whiskers Plot Using ICT
}

\author{
Moeketsi Simon Mosia \\ School of Mathematics, Natural Sciences and Technology Education, \\ University of the Free State, Bloemfontein campus \\ Email: Mosiams@ufs.ac.za
}

\author{
Doi:10.5901/mjss.2014.v5n27p833
}

\begin{abstract}
This study intend to enhance the teaching and learning interpretation of box-and-whisker plot using statistical $R$ environment software. The researcher adopts statistical $R$ software package to enhance the learners understating of box-and-whiskers plot. The researcher explores the beauty of exuberant graphical illustrations that are built in statistical $R$ environment in enhancing the teaching and learning of box-and-whisker plots. The study adopts participatory action research (PAR) approach through the lens of bricolage. The participants are 43 grade 11 mathematics learners and three mathematics teachers and the school principal. The data was generated using free attitude interview after each lesson for learners and teachers to reflect on the lesson. The qualitative data was analysed using critical discourse analysis (CDA). The results of the study report that learners' reflection on the lesson pointed out that, it felt like they were playing. Through the use of statistical $R$ environment the learners where able conceptualise the difference between maximum, minimum and outlier and the interpretation of the box area. Furthermore, the study report that learners' interpretation of box and whiskers improved.
\end{abstract}

Keywords: Statistical R environment, Box-and-Whiskers, Grade 11

\section{Introduction}

This study intend to enhance the teaching and learning interpretation of box-and-whisker plot using ICT. The study explores the use of statistical R environment software package as our ICT tool to enhance the teaching and learning interpretation of box-and-whiskers plots. Box-and-whisker plots (also known as box plot) are statistical technique used to visualise the distributions of data in investigating where the data are centred and how they are distributed over a range of variables(Kortenkamp \& Rolka, 2010). The researcher intends to take the advantage of graphical and data manipulation features built in statistical $\mathrm{R}$ environment to enhance the teaching and learning of interpretation of box-and-whisker plots. In order for learners to be competent in interpreting box-and-whiskers plots, the following concepts are important: quartiles, inter-quartile, median, minimum, range and maximum for any given dataset (Chick \& Pierce, 2013).

However, the Department of Basic Education National Diagnostic Report ( 2011, 2012, 2013) states that learners have the following challenges concerning the box plots: misinterpretation of box and whisker plot; inability to link fivenumber summary and box and whisker plot; Moreover, number of literature studies capture that internationally learners have challenges the following challenges concerning box plots: Learners interpret the lager area of the box as representing more observation; misinterpretation of lower quartile, upper quartile, mean, median; interpreting each value exhibited on the box plot as an observed value (Bekker, Biehler \& Konold, 2004; Pfannkuch, 2006; Lem, Onghena, Verschaffel, \& Van Dooren, in press; Lem, Onghena, Verschaffel, and Van Dooren, 2012, Lem, Onghena, Verschaffel, \& Van Dooren, 2013).

However, literature reports on different strategies that were developed in response to the preceding learners' challenges in interpretation of box-and-whisker plot. However, for the purpose of this study we will devote much time on ICT based teaching strategies. The most popular ICT tools used to teach box and whisker plots are Minitool 1 and 2, and Tinkerplots software packages (Yilmaz, 2013; Lem, Onghena, Verschaffel, \& Van Dooren 2013; Bakker, 2004). In response to learners' misconception of the lager area of the box Lam et al. (2013) used a bottom up approach. This approach refers to learners first see a dot plot, which is then divided into two and later four equally sized parts using Minitool and Tinkerplots. More so, (Kortenkamp \& Rolka, 2010) proposed a new approached that employs an interactive box and whiskers plot applet to enhance the teaching of box and whiskers plot. The results of the study shows that using applet learners could be able to able to realise that box and whiskers plot divided the data set into four parts that contain $25 \%$ of the data set. The study of Lem, Onghena, Verschaffel, \& Van Dooren (2014) shows that learners still major difficulties in interpreting the boxplot. Therefore, the study intends to develop a strategy to in contribution to enhance the 
teaching and learning of box and whiskers plot.

In order to develop the strategy to enhance the teaching and learning of box and whiskers the researcher find it fit to explore the features and conditions that constitute the partial success of the preceding strategies. The attractive features of Minitool 2 and Tinkerplots in teaching box-and-whisker plots is that they allow learners to compare data set with the same range and centre but different spread (Bakker, 2004: 8; Yilmaz, 2013). These software packages enable learners to visualize the individual observation using the Dot plots, which helps learners to interpret box and whisker plots better. Using Minitool 2 and Tinkerplots learners can easily calculate the median of any given data set as follows: Minitool 2 divide data set into two equal groups while with Tinkerplots learners can click on median value button. The preceding feature of these software tools serves as a basis for software choice of the researcher. Therefore, it is on this basis that the researcher proposed Statistical R environment software. Statistical $\mathrm{R}$ environment has seen much success in statistical programming and modelling, and has exuberant graphical illustration features (Seefeld, 2007).

\section{Theoretical Background}

Box and whiskers plot also known as box plot are a graphical statistical technique used to analyse the data (Kortenkamp, Rolka, 2010). Box and whiskers plots gives the analysis ways in which dataset is centred and distributed using five number summary that is: minimum, lower quartile, median, upper quartile, maximum. Figure 1 is an example of a box and whiskers plot constructed with Statistical $\mathrm{R}$ environment software with the dataset $x=3,1,2,3,4,5,7,5,9,15,9$. More so, just like Minitool and Tinkerplot, Statistical R environment software can easily be used to calculate the five number summary using very basic commands such as $\operatorname{mix}(x)=1, \max (x)=15, \operatorname{median}(x)=5$.

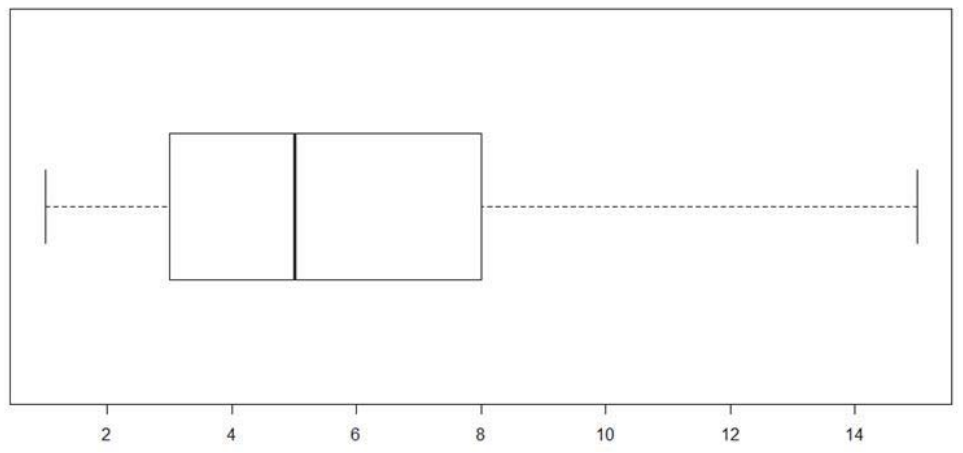

Figure 1. Box and whiskers plot

We find Statistical $\mathrm{R}$ environment better than other software in the sense that its output explains how data is distributed in box and whisker plot. Figure 2 below is the output of Statistical $R$ environment when computing the quartiles. This output help the learner to understand that the quartile divides dataset in percentages. This also help the learner to make a connection between quartiles and box and wishers plot. More so,

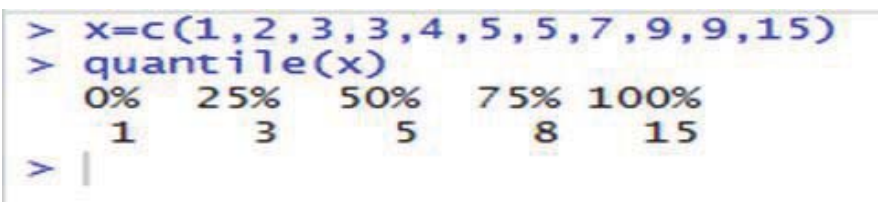

Figure 2. Statistical R command to compute quartiles

The output $Q_{1}=3$ is clearly shows that $Q_{1}$ is the cut off of $25 \%$ of the data, so it is with other quartiles. Statistical R environment would help the learners to make connection between median and quartile two. Moreover, $R$ environment would enhance learners' logical reasoning and meaningful data manipulations than both packages since $\mathrm{R}$ environment provides learners with easy ways to manipulate their dataset by simply altering a vector of data and observe the impact is has on box and whiskers plot (Seefeld, 2007; Everitt \& Hothorn, 2005). Kortenkamp, Rolka (2010) states that of one the challenges of using ICT tools to teach box and whiskers plot is that they use different definition of median to that learners 
use and understand. However, using Statistical $\mathrm{R}$ does not temper with learners understand if a median since it is explicitly shown on the output of the quartile see Figure 2.

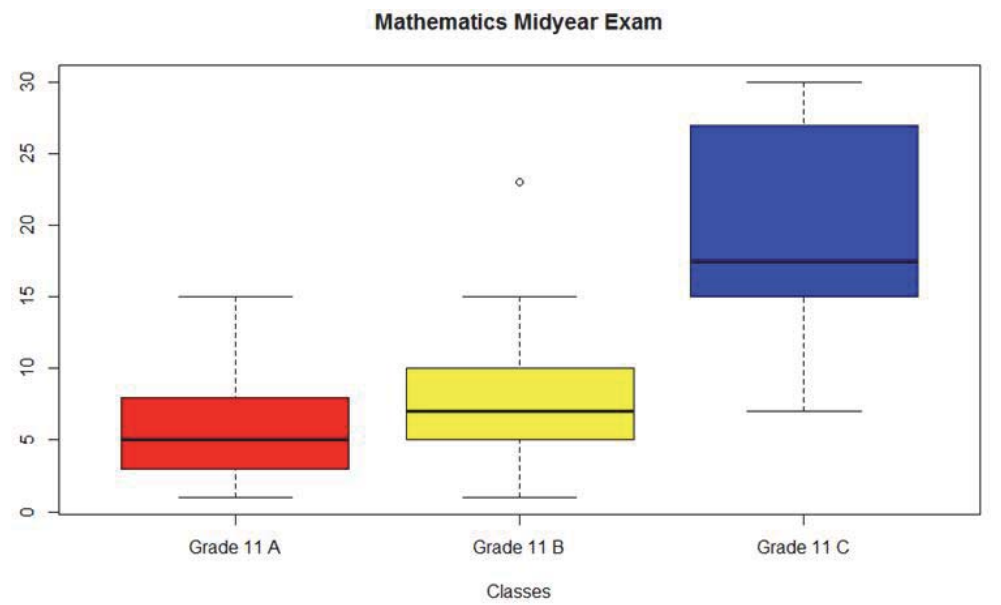

The statistical $\mathrm{R}$ environment provided learners with the opportunities to explore multiple colours in making sense of the information exhibited on the box and wishers plot. Box and whiskers plot are very useful when comparing two datasets (Kader \& Perry, 1996; Mullenex, 1990). Therefore, Statistical R environment enable learners to conceptualise the comparison of different box and whiskers plot through data manipulation. For example, Figure 3 shows that the median of grade $11 \mathrm{~B}$ is higher than that of grade $11 \mathrm{~A}$, therefore learners could manipulate the dataset until the first twenty five percent of grade $11 \mathrm{~A}$ is approximately equal to that of grade $11 \mathrm{~B}$. As the learners are observing the change that occurs as they manipulate the data, it would help them to conceptualise that $50 \%$ of data observed in grade $11 \mathrm{~A}$ is approximately equal to the $25 \%$ of the data observed in grade $11 \mathrm{~B}$ which implies that grade $11 \mathrm{~B}$ is better. Furthermore, that the minimum value observed in grade $11 \mathrm{C}$ is approximately equal to the cut off of $50 \%$ on the data observed in grade $11 \mathrm{~B}$.

\section{Research Design and Research Methodology}

The study would make use of the participatory action research method (PAR). The researcher finds participatory action research (PAR) suitable for designing a strategy to enhance the teaching and learning of interpretation of box and whiskers using Statistical $\mathrm{R}$ environment since it presents learning experience for both researcher and participants (Weber-Pillwax, 2009). According to Weber-Pillwax (2009) PAR brings transformation at three levels, it first starts with us as a team of co-researchers, others and our mutual environment. It is for these reasons that we find this kind of approach to research most relevant and in line with the objective of the study. PAR help us to create a team of co-researcher and create a platform for learners' voice to be heard in the development of a strategy to enhance teaching and learning of interpretation of box and whiskers using Statistical R environment. The learners are to be empowered to "work effectively with others as members of a team, group, organization and community" (DoE, 2003).

The study was conducted in Bloemfontein, Free State province, with 43 grade 11 learners, three mathematics teachers and the school principle. Through the use of PAR we generated data during out planning meeting (audio recordings), class presentation, learners' assessment and lesson reflections (audio recordings). In order to make meaning and capture the voices of all participants at different levels, the study employs critical discourse analysis (CDA). CDA enables us to interpret the spoken words in its context (Van Dijk, 2008)

\section{Findings and Discussion}

In commencement of our meeting with the principal, one of the teachers Mr Ramanthe explained the purpose of our meeting with the principle. The principal started by saying that

..bo ntate bana bana le software e ba e fumaneng, ke sure ke lemo tse tharo jwale but I have never seen them using it....ntate Khama, how is this software different from the one you have already.... (These gentlemen have a software that they received, I am sure it's been three years now but I have never seen them using it... Mr Khama, how is this 
different from the one you already have)

From this extract it seems that mathematics teachers have resources that they have not been using for three years. Moreover, the principal seems to be concerned about resources that are made available but never used. The principal seems to be a good manager of the school since according to him if the teachers were using the software he should have seen them. However, the phrase "it's been three years now" suggests that the principal has been aware for three years that the teachers are not using the software that was intended to enhance their teaching but did nothing about situation. More so, the principal seems to be rejecting the notion of using additional software. One of the teachers $\mathrm{Mr}$ Khama in response to the principal said that

Tata, ke hore fela motho ha a fumane nako... bana bana ba feila (Tata, It is just that a person never gets time ... this leaners are failing)

From the extract the word Tata means Father, in the context of this study we understand that the choice of the word Tata shows how respected even powerful the principal is perceived by Mr Khama. Furthermore, power relations are evident when the principal blames the teachers for not using the software for three years, but never held himself accountable as the manager. The phrase it is just that a person never gets time suggest that Mr Khama is of the opinion that when he has time it will be too easy for him to use the available software to teacher. Furthermore, it seems that $\mathrm{Mr}$ Khama is concerned about the leaners failing mathematics such that he never gets time to use the software to teach mathematics. Oldfiel \& Futurelab (2010) study reports that investing in ICT does not guarantee effective use or what impact it will have. Moreover, their study states that in 6 countries a significantly large amount of teachers find ICT not providing any benefits for teaching and learning. This have become evident in Mr Khama conversation, it seems he does not perceive the available software as a means to enhance his teaching method but rather a waste of time. This is also evident when Mr Khama says that learners are failing that is why he cannot use the software. However, this also suggests that Mr Khama, might not have the regarded ICT pedagogical content knowledge to teach mathematics using the available software. According to Cavas, Cavas, Karaoglan \& Kisla (2009) ICT must be seen as a media for learning, discovering, sharing and creating knowledge and it seems that Mr Khama does not see it in this light.

However, the meeting resulted in designing a research planning to response to learners' challenges of interpreting box and whiskers plot and teachers' challenges to use ICT with special reference to Statistical R environment. Given the participants lack of exposure to computers, we spend a week teaching the participants basic computer skills including basic commends in Statistical $\mathrm{R}$ software environment package. According to mathematics Further Education and Training (FET) Curriculum Assessment Policy Statement (CAPS) learners must be able to collect, organise and analysis the data. A problem based approach was employed and Learners were given min-research project in groups of five to investigate learners' absenteeism as this was one of the school's challenges in grade 9 class. The process of investigations empower learners with research skills, data collection methods, and exposure to applications of statistics in real life problem solving. One of the teachers Mr Ramanthe, in reflection to the use of Statistical R environment state that

Software yena (This software) helps them (learners) to quickly perform the calculations so that they can focus on the interpretation of the results since these is our major concern.

From this extract, it seems that Mr Ramanthe starts to see the importance of using ICT with special reference to Statistical $\mathrm{R}$ environment software package. Mr Ramanthe highlights that $\mathrm{R}$ environment helps the learners to do computation quickly This is made possible by using $\mathrm{R}$ command such as $\min$ (of data) $=1$; $\max$ (of data) $=25$; median (of data) $=5$; for a given set of data 2, 1, 4, 25, 5, 3, 3, 7, 9, 15, 9, 5 . One notable fact is that the participants were able to discover some of the commands with guidance. This is explained by the theory of Vygotsky that a teacher becomes More Knowledgeable Other (MKO), in guiding the learners to discover other Statistical R environment commands (Mwewa, Boby \& Musonda, 2012).

\section{Learners' Interpretation of Box and Whiskers Plots}

This section aims to facilitate the discussion on learners' interpretation of box and whiskers plots on their project findings. After 2 weeks of computer literacy and Statistical R environment commands needed for analysing data using in $\mathrm{R}$, followed by 2 weeks of how to use $\mathrm{R}$ to enhance the teaching and learning interpretations, learners summited their statistical analysis report project. From the learners report $76.13 \%$ of the groups' reports showed a great improvement of leaners interpretation of the median, learners interpreted median as "the value of the observation at the middle of the data". This was a great improvement since it helped the learners to see the median in the light of quartile two. The 
possible cause is that Statistical $\mathrm{R}$ environment helped learners visualise quartiles together with their percentage spread see Figure 2. As a result, this helped the learners remedy a challenge reported by Lem, Onghena, Verschaffel, \& Van Dooren (2013) in their study that learners interpret median as an independent of the whole dataset. However, languages was found to be the barrier for learners to express their findings. This was found evident when results of some groups' interpretation were not discuss but conclusion was made based on intervals, for example

"Class A: Interval between min and Q1 is 2

- Interval between $\mathrm{Q} 1$ and $\mathrm{Q} 2$ is 2

- Interval between Q2 and Q3 is 4

- Interval between $\mathrm{Q} 3$ and max is 6

Class B: Interval between min and Q1 is 4

- Interval between $\mathrm{Q} 1$ and $\mathrm{Q} 2$ is 2

- Interval between $\mathrm{Q} 2$ and $\mathrm{Q} 3$ is 3

- Interval between $\mathrm{Q} 3$ and $\max$ is 5

Therefore class B have too much absenteeism"

From this group's interpretation of box and whiskers plots after their analysis in statistical $\mathrm{R}$ environment, it seems that they compared the box and whiskers plots of their dataset over the four intervals as shown in Statistical $\mathrm{R}$ environment output. This could be credited to Statistical $\mathrm{R}$ environment that learners compare box and whiskers plots over four interval given by five number summary on the plots. However, this example still shows that some leaners do not understand this intervals as representation of $25 \%$ of dataset.

\section{Lesson Reflections}

Learners were requested to reflect on their experiences in learning with Statistical $\mathrm{R}$ software. One of the Learners Palesa says that

...ntate ho etsa mmetse ka computer e kare a se mmetse man! ...o monate ... ekare wa sebetsa.(Sir doing mathematics using the computer it does not seem like doing mathematics....it is enjoyable, it's like I am working)

From the extract it seems like Palesa is not enjoying doing mathematics, however, with the use of statistical $\mathrm{R}$ environment software package Palesa seems to be finding mathematics as an enjoyable subject. What Palesa is saying is also captured by Kearney (2010) that the use of ICT increases the learners' interest in mathematics. Furthermore, the studies report that ICT increases the learners' motivation to learn and helps the learners to practically conceptualise mathematical concepts (Cunska \& Savicka, 2012). This is also evident in Palesa's utterance when she states that doing mathematics with the computer feels like you are work. Work is this context we understand that it means employed than studying. The Statistical R environment creates a social interaction between learner to learner and learner to teacher which enhanced learners understanding of box and whiskers plot. This is also supported by the Lev vygostky theory that learners' cognitive development is enhanced by social interaction (Mwewa, Boby \& Musonda, 2012).

\section{References}

Cunska, A., \& Savicka, I. (2012). Use of ICT Teaching-Learning Methods make School Math Blossom. Procedia - Social and Behavioral Sciences, 69, 1481-1488.

Dijk, T. A. V. (1997). 18 Critical Discourse Analysis.

Dooren, V., Makar, I. K., \& Sousa, B. D. (2014). ICOTS9 (2014) Contributed Paper - RefereedLem, Kempen, Ceulemans, Onghena, Verschaffel \& Van Dooren. , 9, 7-10.

Fuglestad, A. B. (2003). ICT and mathematics learning (ICTML). , 1-10.

Huckin, T. (2002). Critical Discourse Analysis and the Discourse of Condescension.

Hudson, R., \& Porter, A. (2010). ICT USE TO IMPROVE MATHEMATICS LEARNING IN SECONDARY SCHOOLS.

Kearney, C. (2010). Efforts to Increase Students' Interest in Pursuing Mathematics, Science and Technology Studies and Careers Member Countries.

Kortenkamp, U., \& Rolka, K. (2010). USING TECHNOLOGY IN THE TEACHING AND LEARNING OF BOX PLOTS. , $1070-1080$.

Le Roux, K. (2008). A Critical Discourse Analysis of a Real-World Problem in Mathematics: Looking for Signs of Change. Language and Education, 22 (5), 307-326.

Lim, C. P. (2002). In schools: a proposal. , 33 (4), 411-422.

Paper, C. (2011). A computer-assisted Framework based on a cognitivist learning theory for teaching mathematics in the early primary years, A computer-assisted framework based on a cognitivist learning theory for teaching mathematics. , 39-45. 
Peter, M., Elizabeth, S. B., \& Allan, M. (2014). An Investigation into the Development of ICT Skills among Mathematics Students in Higher Learning Institutions, A Case of Mukuba University. , 3 (7), 257-272.

Reynolds, N. (2006). Analysing classroom interactions using critical discourse analysis. , 5, 273-280.

Roux, K. L. (2008). Relevance and access in undergraduate mathematics: using discourse analysis to study mathematics texts.

Sharon, V. (2012). Discoursing Mathematically: Using Discourse Analysis to Develop a Sociocritical Perspective of Mathematics Education. , 22 (1).

Van Dijk, T. A. (1993). Principles of Critical Discourse Analysis. Discourse \& Society, 4 (2), 249-283.

Verenikina, I. (2009). Vygotsky in Twenty-First-Century Research.

Wodak, R., \& Meyer, M. (2008). Critical Discourse Analysis: History, Agenda, Theory, and Methodology1, 1-33. 